S1 Table. Details and references of ant elevational gradients used in analyses. List of robust and informative elevational gradients in ant species richness. Each denoted by study site (reference), diversity pattern (D: decreasing; LP: low-plateau; MP: mid-peak), data scale (L: local; R: regional), survey methods (B: baited traps, F: canopy or bark fogging; H: hand collection; L: litter sifting; M: malaise traps; P: pitfall traps; R: museum records), latitude, mountain base climate, $\%$ of gradient sampled, meters unsampled at mountain base, mountain height (m), temperature $r^{2}$ values, MDE diversity and range $r^{2}$ values, species-area $r^{2}$ values, best fit EGCM environmental model, EGCM environmental model $r^{2}$ values, EGCM simulation $r^{2}$ values, and total number of species and genera on gradient.

\begin{tabular}{|c|c|c|c|c|c|c|c|c|c|c|c|c|c|c|c|c|}
\hline Study Site (Reference) & $\begin{array}{c}\text { Div. } \\
\text { Pattern }\end{array}$ & Scale & $\begin{array}{l}\text { Survey } \\
\text { Methods }\end{array}$ & Lat. & Climate & $\begin{array}{c}\% \\
\text { Grad. }\end{array}$ & $\begin{array}{l}\text { Unsamp. } \\
\text { base (m) }\end{array}$ & $\underset{\text { (m) }}{\text { Mtn Hgt }}$ & $\underset{r 2}{\text { Temp }}$ & $\begin{array}{l}\text { MDE } \\
\text { Div. } r 2\end{array}$ & $\begin{array}{l}\text { MDE } \\
\text { Rng } r 2\end{array}$ & $\begin{array}{c}\text { Area } \\
r 2\end{array}$ & $\begin{array}{c}\text { EGCM } \\
\text { best mod }\end{array}$ & $\begin{array}{l}\text { EGCM } \\
\text { Env. } r 2\end{array}$ & $\begin{array}{l}\text { EGCM } \\
\text { Sim. } r 2\end{array}$ & $\begin{array}{l}\text { Species } \\
\text { (Genera) }\end{array}$ \\
\hline Soutpansberg, South Africa (Munyai \& Foord 2012) & $\mathrm{D}$ & $\mathrm{L}$ & $\mathrm{P}$ & -23.0 & Arid & 72.3 & 106 & 938 & 0.745 & --- & --- & 0.887 & --- & --- & --- & $78(28)$ \\
\hline Utah, USA (Allred 1982) & MP & $\mathrm{R}$ & $\mathrm{H}$ & 39.5 & Arid & 78.9 & 52 & 3459 & 0.149 & 0.111 & 0.080 & 0.509 & ATP & 0.698 & 0.969 & $121(23)$ \\
\hline Colorado, USA (Gregg 1963) & MP & $\mathrm{R}$ & $\mathrm{H}$ & 39.0 & Arid & 95.1 & 49 & 3367 & 0.492 & 0.162 & 0.027 & 0.809 & AT & 0.326 & 0.978 & $149(40)$ \\
\hline Nevada, USA (Wheeler \& Wheeler 1986) & MP & $\mathrm{R}$ & $\mathrm{H}, \mathrm{R}$ & 38.5 & Arid & 92.8 & 9 & 3835 & 0.000 & 0.896 & 0.174 & 0.900 & $\mathrm{AT}$ & 0.981 & 0.975 & $157(28)$ \\
\hline W. Texas, USA** & MP & $\mathrm{R}$ & $\mathrm{B}, \mathrm{H}, \mathrm{L}, \mathrm{R}$ & 32.8 & Arid & 92.1 & 0 & 2498 & 0.148 & 0.379 & 0.353 & 0.907 & AP & 0.981 & 0.975 & $149(35)$ \\
\hline Chisos Mtns, TX, USA (Van Pelt 1983) & MP & $\mathrm{R}$ & B, H, L, R & 29.3 & Arid & 76.2 & 348 & 1706 & 0.007 & 0.406 & 0.417 & 0.002 & $\mathrm{TP}$ & 0.855 & 0.968 & $81(29)$ \\
\hline Soutpansberg, South Africa (Munyai \& Foord 2012) & MP & $\mathrm{L}$ & $\mathrm{P}$ & -23.0 & Arid & 83.4 & 142 & 1067 & 0.141 & --- & --- & 0.563 & --- & --- & --- & $78(28)$ \\
\hline Cederburg Wilderness, South Africa (Botes et al. 2006) & MP & $\mathrm{L}$ & $\mathrm{P}$ & -32.4 & Arid & 97.8 & 5 & 1964 & 0.000 & --- & --- & 0.004 & --- & --- & --- & $85(24)$ \\
\hline Montenegro (Karaman 2011) & $\mathrm{D}$ & $\mathrm{R}$ & $\mathrm{H}$ & 42.3 & Wet & 77.5 & 100 & 2194 & 0.928 & 0.070 & 0.455 & 0.767 & $\mathrm{~T}$ & 0.794 & 0.283 & $87(28)$ \\
\hline McPherson Range, Australia (Burwell \& Nakamura 2011) & $\mathrm{D}$ & $\mathrm{L}$ & $\mathrm{F}, \mathrm{H}, \mathrm{L}$ & -28.2 & Wet & $66.9^{*}$ & 300 & 1196 & 0.749 & 0.651 & 0.556 & 0.806 & A & 0.777 & 0.970 & $169(57)$ \\
\hline Voralberg, Austria (Glaser 2006) & LP & $\mathrm{R}$ & $\mathrm{R}$ & 47.3 & Wet & $59.6^{*}$ & 12 & 2853 & 0.851 & 0.405 & 0.618 & 0.110 & $\mathrm{~T}$ & 0.909 & 0.197 & $65(17)$ \\
\hline Volcan Barva, Costa Rica (Longino \& Colwell 2011) & LP & $\mathrm{L}$ & $\mathrm{L}$ & 10.3 & Wet & $67.3^{*}$ & 50 & 2897 & 0.826 & 0.711 & 0.880 & 0.487 & $P$ & 0.719 & 0.682 & $401(70)$ \\
\hline Espinhaco, Brazil (Araujo \& Fernandes 2003) & LP & $\mathrm{L}$ & $\mathrm{B}, \mathrm{H}$ & -19.4 & Wet & $56.4^{*}$ & 322 & 1241 & 0.775 & 0.950 & 0.406 & 0.773 & $\mathrm{~T}$ & 0.982 & 0.961 & $39(15)$ \\
\hline South Tirol, Italy (Hellrigl 2003) & MP & $\mathrm{R}$ & $\mathrm{H}, \mathrm{R}$ & 46.8 & Wet & $62.6^{*}$ & 9 & 3674 & 0.029 & 0.068 & 0.203 & 0.194 & $\mathrm{~T}$ & 0.189 & 0.922 & $70(24)$ \\
\hline Smoky Mtns, TN, USA (Lessard et al. 2007) & MP & $\mathrm{L}$ & $\mathrm{L}, \mathrm{M}, \mathrm{P}$ & 35.6 & Wet & 75.4 & 161 & 1807 & 0.701 & 0.102 & 0.625 & 0.845 & ATP & 0.727 & 0.953 & $38(20)$ \\
\hline Mt Isarog, Philippines (Samson et al 1997) & MP & $\mathrm{L}$ & $\mathrm{P}, \mathrm{H}$ & 13.7 & Wet & 75.6 & 250 & 1983 & 0.593 & 0.190 & 0.368 & 0.373 & A & 0.575 & 0.971 & $52(20)$ \\
\hline Western Ghats, India (Sabu et al. 2008) & MP & $\mathrm{L}$ & $\mathrm{L}$ & 11.7 & Wet & $58.1^{*}$ & 300 & 2322 & 0.205 & 0.026 & 0.811 & 0.543 & A & 0.200 & 0.845 & $29(18)$ \\
\hline Bocas del Toro, Panama (Olson 1994) & MP & $\mathrm{L}$ & $\mathrm{L}, \mathrm{P}$ & 8.8 & Wet & $58.1^{*}$ & 300 & 2375 & 0.799 & --- & --- & 0.686 & --- & --- & --- & $196(-)$ \\
\hline Ambohitsitondroina, Madagascar (Fisher 1998) & MP & $\mathrm{L}$ & $\mathrm{L}, \mathrm{P}$ & -15.1 & Wet & 95.5 & 25 & 2053 & 0.506 & 0.177 & 0.067 & 0.684 & A & 0.349 & 0.988 & $272(28)$ \\
\hline Fiji (Sarnat \& Economo 2012) & MP & $\mathrm{R}$ & ALL & -17.7 & Wet & 98.1 & 0 & 1324 & 0.654 & 0.359 & 0.096 & 0.850 & A & 0.579 & 0.914 & $174(42)$ \\
\hline
\end{tabular}

$*<70 \%$ of gradient sampled, but primarily upper elevations where diversity had already decreased monotonically

** Moody \& Francke 1982; Cockendolpher \& Francke 1990 


\section{S1: References}

1. Allred DM. Ants of Utah. Gt Basin Nat. 1982;42: 415-511.

2. Araújo LLM, Fernandes GWG. Altitudinal patterns in a tropical ant assemblage and variation in species richness between habitats. Lundiana. 2003;4: 103-109.

3. Botes A, McGeoch MA, Robertson HG, van Niekerk A, Davids HP, Chown SL. Ants, altitude and change in the northern Cape Floristic Region. J Biogeogr. 2006;33: 71-90. doi:10.1111/j.1365-2699.2005.01336.x

4. Burwell CJ, Nakamura A. Distribution of ant species along an altitudinal transect in continuous rainforest in subtropical Queensland, Australia. Mem Queensl Museum. 2011;55: 391-412.

5. Cokendolpher JC, Francke OF. The ants (Hymenoptera, Formicidae) of western Texas. Part II. Subfamilies Ecitoninae, Ponerinae, Pseudomyrmecinae, Dolichoderinae, and Formicinae. Spec Publ Mus Tex Tech Univ. Lubbock, TX: Texas Tech Press; 1990.

6. Fisher BL. Ant diversity patterns along an elevational gradient in the Réserve Spéciale d'Anjanaharibe-Sud and on the western Masoala Peninsula, Madagascar. Fieldiana Zool. 1998;90: 39-67.

7. Glaser F. Biogeography, diversity, and vertical distribution of ants (Hymenoptera: Formicidae) in Vorarlberg, Austria. Myrmecological News. 2006;8: 263-270.

8. Gregg RE. The Ants of Colorado. Univ. of Colorado Press. Boulder, Colorado, xvi. Denver, CO: University of Colorado Press; 1963.

9. Hellrigl K. Faunistik der Ameisen und Wildbienen Südtirols (Hymenoptera: Formicidae et Apoidea). Gredleriana. 2003;3: 143-208.

10. Karaman MG. Zoogeography, diversity and altitudinal distribution of ants (Hymenoptera: Formicidae) in the Mediterranean and the oro-Mediterranean parts of Montenegro. North West J Zool. 2011;7: 26-34.

11. Lessard JJP, Dunn RRR, Parker CCR, Sanders NNJ. Rarity and diversity in forest ant assemblages of Great Smoky Mountains National Park. Southeast Nat. 2007;0: 215-228.

12. Longino JT, Colwell RK. Density compensation, species composition, and richness of ants on a neotropical elevational gradient. Ecosphere. 2011;2: art29. doi:10.1890/ES10-00200.1

13. Moody JV, Francke OF. The ants (Hymenoptera, Formicidae) of western Texas Part I. Subfamily Myrmicinae. Lubbock, TX: Texas Tech Press; 1982.

14. Munyai TC, Foord SH. Ants on a mountain: spatial, environmental and habitat associations along an altitudinal transect in a centre of endemism. J Insect Conserv. 2012;16: 677-695. doi:10.1007/s10841-011-9449-9

15. Olson DM. The distribution of leaf litter invertebrates along a neotropical altitudinal gradient. J Trop Ecol. 1994;10: 129-150.

16. Sabu TK, Vineesh P, Vinod K. Diversity of forest litter-inhabiting ants along elevations in the Wayanad region of the Western Ghats. J Insect Sci. 2008;8: 1-14.

17. Samson DA, Rickart EA, Gonzales PC. Ant diversity and abundance along an elevational gradient in the Phillippines. Biotropica. 1997;29: 349-363.

18. Sarnat E, Economo E. The ants of Fiji. Berkeley and Los Angeles, CA: UC Publications in Entomology, University of California Press; 2012.

19. Van Pelt A. Southwestern Association of Naturalists Ants of the Chisos Mountains, Texas (Hymenoptera: Formicidae). Southwest Nat. 1983;28: 137-142.

20. Wheeler G, Wheeler J. The ants of Nevada. Los Angeles, CA: Natural History Museum of Los Angeles County; 1986. 